\title{
Early clinical outcomes of total thoracoscopic aortic valve replacement
}

\author{
Chao Song ${ }^{1}$, Shengli Jiang ${ }^{2}$, Siming Zhu ${ }^{2}$, and YunLong Fan ${ }^{1}$ \\ ${ }^{1}$ Medical School of Chinese PLA \\ ${ }^{2}$ Chinese PLA General Hospital
}

September 25, 2021

\begin{abstract}
Background: With the promotion of minimally invasive concepts and advances in total thoracoscopic valve surgery, total thoracoscopic aortic valve surgery has become a new option for patients with aortic valve lesions. However, due to its anatomical characteristics, poor surgical field exposure and limited operating space, only a few centers have performed further studies on this procedure. Methods: We evaluate the safety and advantages of total thoracoscopic aortic valve replacement compared to the upper mini-sternotomy AVR group and the conventional AVR group with important perioperative data as well as early postoperative outcomes. Results: All patients successfully underwent elective surgery, with no intraoperative conversion or death occurring. Patients in the total thoracoscopy group had significantly prolonged CPB and aortic clamping (AC) times compared to the other two groups. The average Postoperative chest drainage in the first $24 \mathrm{~h}$ of the total thoracoscopic group was significantly less than the other two groups. The mean VAS pain score in the total thoracoscopic group was significantly less than the other two groups. In addition, the total thoracoscopic group had a significantly decreased ICU stay as well as the total hospital stay. Although the length of mechanical ventilation between groups did not show statistically significant differences, mechanical ventilation in the total thoracoscopy group had a smaller relative number. Conclusions: Despite the need for improvement, total thoracoscopic aortic valve replacement is safe, and may improve clinical outcome
\end{abstract}

Early clinical outcomes of total thoracoscopic aortic valve replacement

Chao Song ${ }^{1,2}$, Yunlong Fan ${ }^{1,2}$, Siming Zhu ${ }^{1,2}$, Shengli Jiang ${ }^{2 *}$

${ }^{1}$ Medical School of Chinese PLA, Beijing, 100853, P.R. China

${ }^{2}$ Department of Cardiovascular Surgery, the First Medical Centre of Chinese PLA General Hospital, Beijing 100853, China

*Correspondence: Dr. Shengli Jiang, Department of Cardiovascular Surgery, Chinese PLA General Hospital, 100853, Beijing, China. E-mail: JiangSL301hospital@163.com.

Conflicts of interest

The authors declare that there are no conflicts of interest.

This research did not receive any specific grant from funding agencies in the public, commercial, or not-forprofit sectors.

Objective: To evaluate the safety and reliability of total thoracoscopic aortic valve replacement with a controlled study and to analyze the current challenges that may need to be addressed.

Background: With the promotion of minimally invasive concepts and advances in total thoracoscopic valve surgery, total thoracoscopic aortic valve surgery has become a new option for patients with aortic valve 
lesions. However, due to its anatomical characteristics, poor surgical field exposure and limited operating space, only a few centers have performed further studies on this procedure.

Methods: A total of 230 patients underwent AVR were divided into the total thoracoscopic AVR group $(\mathrm{n}=18)$, the upper mini-sternotomy AVR group $(\mathrm{n}=43)$, and the conventional AVR group $(\mathrm{n}=169)$ according to the surgical access. Cardiopulmonary bypass (CPB) time, aortic clamping (AC) time, postoperative chest drainage in the first $24 \mathrm{~h}$ (ML), ICU and Hospital stay, Mechanical ventilation length and VAS pain score were used as evaluation variables to evaluate the safety and reliability of total thoracoscopic aortic valve replacement compared to the upper mini-sternotomy AVR group and the conventional AVR group.

Results: All patients successfully underwent elective surgery, with no intraoperative conversion or death occurring. Patients in the total thoracoscopy group had significantly prolonged CPB and aortic clamping (AC) times compared to the other two groups. The average Postoperative chest drainage in the first 24 $\mathrm{h}$ of the total thoracoscopic group was significantly less than the other two groups. The mean VAS pain score in the total thoracoscopic group was significantly less than the other two groups. In addition, the total thoracoscopic group had a significantly decreased ICU stay as well as the total hospital stay. Although the length of mechanical ventilation between groups did not show statistically significant differences, mechanical ventilation in the total thoracoscopy group had a smaller relative number.

Conclusions: Despite the need for improvement, total thoracoscopic aortic valve replacement is safe, and may improve clinical outcome.

\section{Introduction}

Minimally invasive cardiac surgery has become a new trend in modern medicine because of its reduced trauma, less bleeding and shorter post-operative recovery time, and there has been a significant increase in the number of studies related to minimally invasive valve surgery ${ }^{[1]}$. With the promotion of minimally invasive concepts and advances in total thoracoscopic valve surgery, total thoracoscopic valve surgery is not limited to the mitral valve procedure anymore, and total thoracoscopic aortic valve surgery has become a new option for patients with aortic valve lesions. However, due to its anatomical characteristics, poor surgical field exposure and limited operating space, total thoracoscopic aortic valve surgery has not become a routine choice of minimally invasive aortic valve surgery just like total thoracoscopic mitral valve surgery. Total thoracoscopic aortic valve replacement (AVR) was first proposed in 2014 by Vola et al ${ }^{[2]}$, and a single-arm study that included 14 patients who underwent total thoracoscopic AVR in 2016 confirmed the safety and reliability of this procedure ${ }^{[3]}$.

In this study, patients hospitalized for aortic valve lesions who underwent AVR were divided into three groups and underwent conventional AVR, upper mini-sternotomy AVR and total thoracoscopic AVR respectively. Baseline data, perioperative data, and early outcomes were used for observation and comparison to assess the safety and reliability of total thoracoscopic AVR.

\section{Methods}

\section{Patient characteristics}

Between January 2017 and July 2021, a total of 230 patients ( 157 males; mean age $53.9 \pm 8.8$ years) underwent AVR in our department. The underlying pathology leading to the procedure was severe aortic insufficiency due to annulus dilatation/leaflet restriction or severe stenosis due to calcification. All AVRs were completed by the same experienced surgeon, and the access was achieved through one of a thoracoscopic approach, a $\mathrm{r}$ upper mini-sternotomy, and conventional median sternotomy. All patients were divided into the total thoracoscopic AVR group ( $\mathrm{n}=18)$, the upper mini-sternotomy AVR group $(\mathrm{n}=43)$, and the conventional AVR group $(\mathrm{n}=169)$ according to the surgical access. Ethical approval for this retrospective study was granted by the local ethics committee. Individual patient consent was not required.

\section{Surgical Technique}


All patients were evaluated preoperatively for peripheral vascular status by aorto-iliac and femoral artery ultrasonography. The patient was placed in a $30^{\circ}$ left side position, double-lumen endotracheal tubes were placed in all patients. Arterial pressure monitoring was performed via the left radial artery, and the floating catheter was placed through the right internal jugular vein. Femoral arterial and vein cannulations were directly performed using the Seldinger technique with two 5/0 Prolene purse strings through a groin incision $(2.5 \mathrm{~cm})$. Cardiopulmonary bypass $(\mathrm{CPB})$ was routinely established through the femoral artery, femoral vein, and right internal jugular vein. The main surgical hole (approximately $4.5 \mathrm{~cm}$ ) was established in the third intercostal, through which the cardiac arrest fluid flushing tube was also passed. The thoracoscopy was placed through anterior axillary line of the second intercostal space, after inserting the aortic root cannula, while the left cardiac drainage and Chitwood clamp were punctured through the fourth intercostal space, and an artificial pneumothorax is routinely established using $\mathrm{CO} 2$.

\section{Follow-up}

All patients were examined by Transthoracic Echocardiogram (TTE) to evaluate the recovery of cardiac function one week after operation. All discharged patients are followed up at 1 month, 3 months, 6 months and 1 year in our outpatient clinic or by telephone call. Follow-up data include TTE, survival rate, readmission rate, and incidence of adverse events. Follow-up data include TTE, readmission rates, and the incidence of adverse events.

\section{Statistical Analysis}

Perioperative data were analyzed using SPSS 26.0 software (SPSS Inc., Chicago, Illinois, USA). Continuous variables were expressed as means and standard deviations. Categorical variables were expressed as percentages. Continuous variables were compared using Student t-test, ANOVA analysis or non-parametric tests, and categorical variables were analyzed using chi-square test and Fischer's exact test. And the significant differences were defined at $\mathrm{p}<0.05$.

\section{Results}

\section{Operation and postoperative data}

Clinical baseline data of the patients were present in Table 1. No statistically significant differences were observed in the preoperative baseline data, baseline data for the three groups of patients were statistically comparable.

All patients completed the surgery successfully, with no intraoperative death or conversion of surgical procedures. Operation and postoperative characteristics are summarized in Table 2. Patients in the total thoracoscopy group had significantly prolonged $\mathrm{CPB}$ and aortic clamping (AC) times compared to the other two groups. The average Postoperative chest drainage in the first $24 \mathrm{~h}$ of the total thoracoscopic group was 172.22 \pm 56.35 , which was significantly less than the other two groups. The mean VAS pain score in the total thoracoscopic group was $2.33 \pm 0.49$, significantly less than the other two groups which were $2.58 \pm 0.54$ and $3.28 \pm 0.52$ respectively. In addition, the total thoracoscopic group had a significantly decreased ICU stay as well as the total hospital stay, $1.46 \pm 0.27$ and $11.78 \pm 1.26$, respectively. Although the length of mechanical ventilation between groups did not show statistically significant differences, mechanical ventilation in the total thoracoscopy group had a smaller relative number.

As shown in the Table3, none of the patients experienced hospital mortality, serious complications, respiratory failure, secondary intubation, pleural effusion, pneumothorax, cerebral hemorrhage, pulmonary Infection, and sternal dehiscence. Intra-aortic balloon pump (IABP) implantation was performed in two of the patients for transient decreased cardiac output, and the IABP was successfully removed. Delayed extubation (longer than 24h) was observed in two patients in the conventional AVR group due to hypoxemia. Two patient underwent dialysis for renal insufficiency, and this patient had a preoperative history of renal insufficiency. Poor healing of incision was observed in two patients in the conventional AVR group, one with a history of diabetes mellitus and unstable blood glucose levels and the other with a local infection due to sweating. And the intraoperative hypoperfusion may have exacerbated the renal impairment, one patient experienced 
brain infarction without disability. In addition, one perivalvular leak was observed in one patient in the total thoracoscopy group during the 3-month follow-up without cardiac insufficiency as well as hemolytic manifestations and is continuing at follow-up.

\section{Discussion}

In recent years, significant improvements have been made in minimally invasive aortic valve surgery, including direct right mini-incision ${ }^{[4]}$ and thoracoscopic-assisted right mini-incision ${ }^{[5]}$, and robotic-assisted aortic valve surgery ${ }^{[6]}$, all of which are characterized by less trauma, less bleeding, and better recovery compared with conventional aortic valve surgery ${ }^{[7]}$. With similar repair results, total thoracoscopic AVR can also be used for complex valvular lesions, with the advantage of minimally invasive valve surgery. The upper mini-sternotomy group had significantly reduced $24 \mathrm{~h}$ chest drainage, reduced pain, decreased ICU stay and hospital stay when compared to conventional AVR, which was consistent with the previous findings of Totaro $\mathrm{P}$ et al. ${ }^{[8]}$ and Paparella D et al ${ }^{[4]}$. Furthermore, total thoracoscopic AVR had more favorable perioperative assessment data compared to the upper mini-sternotomy group, in except for a prolonged CPB as well as AC time. However, a study by Tokoro $\mathrm{M}$ et al. indicated that all-thoracoscopic AVR had similar CPB and AC times compared to conventional AVR ${ }^{[9]}$. Cresce GD et al. showed that the type of valve may have a significant effect on $\mathrm{CPB}$ and $\mathrm{AC}$ time, and we were failed to perform a subgroup analysis because of the insufficient number of procedures ${ }^{[10]}$. In addition, since Vola et al. first reported total thoracoscopic AVR in 2014, only a few centers have performed further studies on this procedure. We think that factors including the anatomical characteristics of the patient's aorta, the insufficient number of aortic valve procedures performed by the surgeon, and the insufficient exposure of the surgical field by the assistant during the procedure limit the development of total thoracoscopic AVR procedures.

The first is patient selection based on the anatomical characteristics of the aortic valve structure, in our experience, a wider sinotubular junction region may be more favorable for thoracoscopic exploration, and a narrower junction region may result in poorer surgical field of view exposure. The severity of calcification of the diseased aortic valve may also render total thoracoscopic AVR more difficult, leading to slightly unfavorable surgical outcomes. In addition, the height of the aortic valve position may be another factor affecting the exposure of the surgical field. In contrast, the conventional AVR, upper mini-sternotomy AVR, is less demanding on the anatomical characteristics of the aortic valve and is generally applicable. Technical improvements in thoracoscopic devices may be a viable solution in the future.

The second is the limitation on the number of heart valve procedures the surgeon has completed. Based on the valve surgery learning curve assessment, after completing a sufficient number of right heart valve procedures, one can attempt mitral valve surgery and subsequently aortic valve surgery ${ }^{[11]}$, which is also a process of re-familiarization with the cardiac anatomy. Our previous study identified the safety as well as reliability of total thoracoscopic mitral valve repair with superior early postoperative outcomes, with the exception of prolonged AC and CPB times ${ }^{[12]}$. Similarly, the increased AC and CPB times are reasonable due to the limitations of the aortic valve anatomic region as well as the sinotubular junction region. Our study identified that total thoracoscopic MVP provided equally satisfactory surgical results compared to conventional MVP and that stabilization could be achieved gradually after completion of the 75th procedure, which was similar to the results of previous learning curve studies of minimally invasive valve surgery. Although there were significant differences between different studies, the overall tendency of change in CUSUM was similar, with the number of total thoracoscopic MVPs required to overcome the learning curve ranging from 64 to $116^{[13-15]}$. However, we failed to perform an assessment of the learning curve of CUSUM (Cumulative Sum) based on total thoracoscopic AVR due to the insufficient number.

The third is the insufficient cooperation between the surgeon and the assistant, decreased cooperation will directly lead to inferior surgical field exposure, which in turn will affect suturing and knotting results.

It maybe noteworthy that one case of perivalvular leak was observed in the total thoracoscopic group at the 3 -month follow-up, as the incidence of perivalvular leakage after AVR was less than 0.01 in our center, this may be the result of inferior suture angles and weak knotting. 


\section{Strengths and limitations}

The advantage of this study compared with previous studies is that we conducted a controlled study of total thoracoscopic AVR, upper mini-sternotomy AVR and conventional AVR, and the conclusions of the study was more reliable than previous single-arm studies and the occurrence of early postoperative adverse events was also analyzed.

This study also has some limitations. Firstly, this study is a retrospective study, and the selection of patients undergoing total thoracoscopic AVR may have undergone operator intervention. Second, the statistical results may be biased due to the insufficient number of total thoracoscopic AVRs. Finally, some of the study subjects are still in the process of follow-up, and we will continue to follow up in future studies.

\section{Conclusion}

Total thoracoscopic AVR had prolonged CPB and AC times compared to upper mini-sternotomy AVR and conventional AVR, with significant advantages in postoperative 24-hour drainage, ICU and total length of stay, and VAS pain scores, and postoperative perivalvular leakage may be a challenge that needs to be addressed at this time.

\section{References}

[1] Schmitto JD, Mokashi SA, Cohn LH. Minimally-invasive valve surgery. J Am Coll Cardiol. 2010. 56(6): $455-62$.

[2] Vola M, Fuzellier JF, Chavent B, Duprey A. First human totally endoscopic aortic valve replacement: an early report. J Thorac Cardiovasc Surg. 2014. 147(3): 1091-3.

[3] Vola M, Fuzellier JF, Campisi S, et al. Closed chest human aortic valve removal and replacement: Technical feasibility and one year follow-up. Int J Cardiol. 2016. 211: 71-8.

[4] Paparella D, Malvindi PG, Santarpino G, et al. Full sternotomy and minimal access approaches for surgical aortic valve replacement: a multicentre propensity-matched study. Eur J Cardiothorac Surg. 2020. 57(4): 709-716.

[5] Vola M, Campisi S, Anselmi A, Faure M, Fuzellier JF, Gerbay A. Video-assisted minithoracotomy approach: technical developments towards totally endoscopic sutureless aortic valve replacement. J Card Surg. 2014. 29(4): 494-6.

[6] Edelman JJ, Thourani VH. Commentary: Robotic aortic valve replacement-fad or future. J Thorac Cardiovasc Surg. 2021. 161(5): 1763-1764.

[7] Gumus F, Hasde AI, Bermede O, Kilickap M, Durdu MS. Multiple Valve Implantation Through a Minimally Invasive Approach: Comparison of Standard Median Sternotomy and Right Anterior Thoracotomy. Heart Lung Circ. 2020. 29(9): 1418-1423.

[8] Totaro P, Carlini S, Pozzi M, et al. Minimally invasive approach for complex cardiac surgery procedures. Ann Thorac Surg. 2009. 88(2): 462-6; discussion 467.

[9] Tokoro M, Sawaki S, Ozeki T, Orii M, Usui A, Ito T. Totally endoscopic aortic valve replacement via an anterolateral approach using a standard prosthesis. Interact Cardiovasc Thorac Surg. 2020. 30(3): 424-430.

[10] Cresce GD, Sella M, Hinna Danesi T, Favaro A, Salvador L. Minimally Invasive Endoscopic Aortic Valve Replacement: Operative Results. Semin Thorac Cardiovasc Surg. 2020. 32(3): 416-423.

[11] McKay R, Ross DN. Primary repair and autotransplantation of cardiac valves. Annu Rev Med. 1993. 44: 181-8.

[12] Cui H, Zhang L, Wei S, et al. Early clinical outcomes of thoracoscopic mitral valvuloplasty: a clinical experience of 100 consecutive cases. Cardiovasc Diagn Ther. 2020. 10(4): 841-848. 
[13] Hayashi Y, Nakamura Y, Hirano T, Ito Y, Watanabe T. Cumulative sum analysis for the learning curve of minimally invasive mitral valve repair. Heart Vessels. 2021. 36(10): 1584-1590.

[14] Dokollari A, Cameli M, Kalra DS, et al. Learning curve predictors for minimally invasive mitral valve surgery; how far should the rabbit hole go. J Card Surg. 2020. 35(11): 2934-2942.

[15] Vo AT, Nguyen DH, Van Hoang S, et al. Learning curve in minimally invasive mitral valve surgery: a single-center experience. J Cardiothorac Surg. 2019. 14(1): 213.

Table1 Preoperative baseline characteristics

\begin{tabular}{llll}
\hline Variable & Total thoracoscopic AVR & upper mini-sternotomy AVR & conventional A \\
\hline Male/Female & $13 / 5$ & $29 / 14$ & $115 / 54$ \\
Age & $53.83 \pm 8.6$ & $53.3 \pm 11.03$ & $54.1 \pm 8.2$ \\
Weight & $68.83 \pm 16.64$ & $69.49 \pm 13.12$ & $65.58 \pm 11.34$ \\
Height & $165.56 \pm 12.65$ & $166.63 \pm 9.94$ & $164.87 \pm 8.03$ \\
Hypertension & $5 / 18$ & $15 / 43$ & $55 / 114$ \\
Hypohepatia & 0 & 0 & 2 \\
Renal insufficiency & 0 & 1 & 3 \\
Diabetes & $2 / 16$ & $5 / 43$ & $13 / 145$ \\
BMI & $24.8 \pm 3.74$ & $24.7 \pm 3.66$ & $25.16 \pm 3.69$ \\
CAD & $1 / 19$ & $3 / 43$ & $13 / 145$ \\
AF & $2 / 18$ & $4 / 43$ & $11 / 147$ \\
EuroScore II & $2.685 \pm 3.99$ & $2.55 \pm 2.04$ & $2.31 \pm 1.98$ \\
Echocardiographic Examination (on admission) & & & $36.35 \pm 4.66$ \\
Left atrial diameter (LA) & $37.5 \pm 5.8$ & $36.05 \pm 6.01$ & $54.97 \pm 5.9$ \\
Left ventricular end-diastolic diameter (LVDD) & $56.72 \pm 7.71$ & $55.23 \pm 8.05$ & $59.76 \pm 11.1$ \\
Left ventricular ejection fraction (LVEF) & $62.44 \pm 7.2$ & $63.33 \pm 8.65$ & $102 / 169$ \\
NYHA CLASS III/IV & $10 / 18$ & $27 / 43$ & \\
\hline
\end{tabular}

Table2 Operation and postoperative characteristics

\begin{tabular}{llll}
\hline Variable & Total thoracoscopic AVR & upper mini-sternotomy AVR & convention \\
\hline CPB (min) & $128.89 \pm 28.24$ & $102.88 \pm 29.76$ & $99.29 \pm 39.0$ \\
AC (min) & $99.39 \pm 29.08$ & $76.05 \pm 22.57$ & $71.01 \pm 32.9$ \\
Postoperative chest drainage in the first $24 \mathrm{~h}(\mathrm{ML})$ & $172.22 \pm 56.35$ & $261.16 \pm 104.6$ & $368.11 \pm 14$ \\
ICU stay & $1.46 \pm 0.27$ & $1.93 \pm 0.44$ & $2.18 \pm 1.36$ \\
Hospital stay (D) & $11.78 \pm 1.26$ & $13.05 \pm 1.43$ & $13.81 \pm 1.85$ \\
Mechanical ventilation length (H) & $11.8 \pm 3.84$ & $13.65 \pm 7.75$ & $14.02 \pm 5.86$ \\
VAS & $2.33 \pm 0.49$ & $2.58 \pm 0.54$ & $3.28 \pm 0.52$ \\
Valve size & $22.67 \pm 1.03$ & $23.18 \pm 1.79$ & $23.07 \pm 1.64$ \\
\hline
\end{tabular}

Table3 Postoperative adverse event

\begin{tabular}{llll}
\hline Variable & Total thoracoscopic AVR & upper mini-sternotomy AVR & conventional AVR \\
\hline Hospital mortality, n (\%) & 0 & 0 & 0 \\
Serious complications & 0 & 0 & 0 \\
Secondary thoracotomy & 0 & 0 & 0 \\
Respiratory failure, n (\%) & 0 & 0 & 0 \\
IABP implantation & 0 & 0 & 2
\end{tabular}




\begin{tabular}{llll}
\hline Variable & Total thoracoscopic AVR & upper mini-sternotomy AVR & conventional AVR \\
\hline Delayed extubation & 0 & 0 & 2 \\
Secondary intubation & 0 & 0 & 0 \\
Dialysis for Renal Failure & 0 & 0 & 2 \\
Pleural effusion & 0 & 0 & 0 \\
Pneumothorax & 0 & 0 & 0 \\
Cerebral infarction & 0 & 0 & 1 \\
Cerebral hemorrhage & 0 & 0 & 0 \\
Pulmonary Infection & 0 & 0 & 0 \\
Poor healing of incision & 0 & 0 & 2 \\
Sternal dehiscence & 0 & 0 & 0 \\
Perivalvular leakage & 1 & 0 & 1 \\
Readmission & 0 & 0 & 0 \\
\hline
\end{tabular}

